\title{
Triangular Fibrocartilage Complex (TFCC)
}

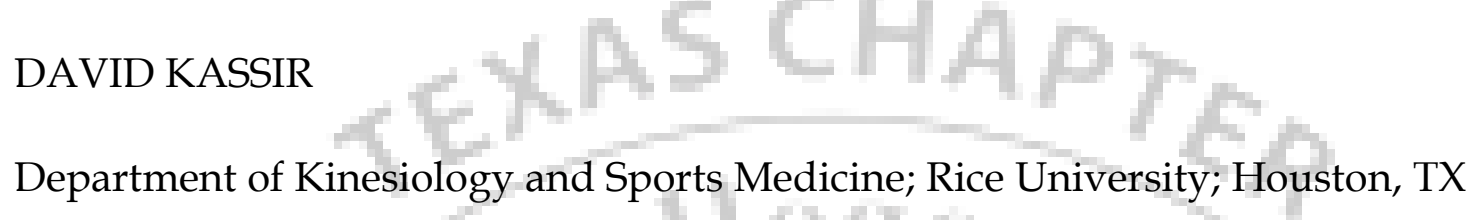

Department of Kinesiology and Sports Medicine; Rice University; Houston, IX

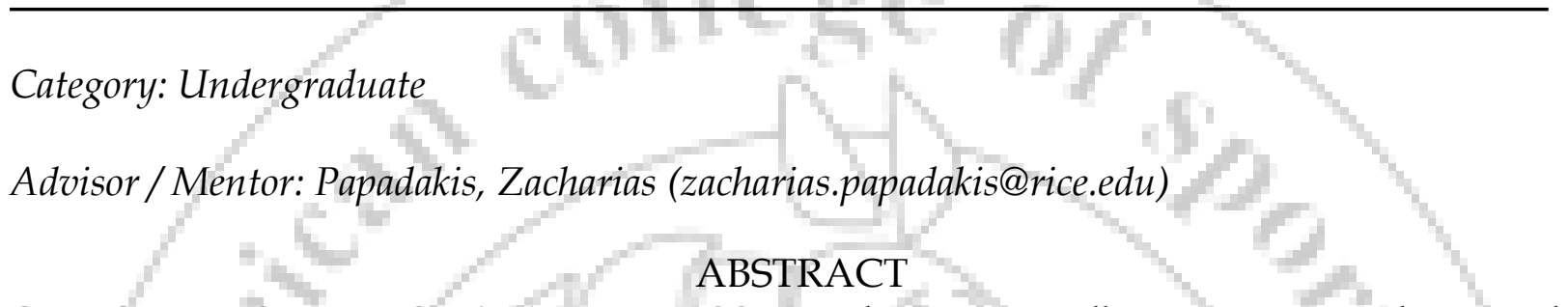

CLINICAL PRESENTATION \& EXAM: In TFCC cases, the patient usually reports injury to the triangular fibrocartilage and pain on the ulnar side of the wrist. This pain is associated to a specific occurrence of falling on an outstretched hand, from a forearm hyper rotation or an axial load and distraction injury to the ulnar boarder of the forearm. This type of falling and acute injury is common in many sports. Pain is especially pronounced when loading the wrist in extension or when supinating/pronating the wrist. In contrast, loading the wrist in a neutral position does not tend to cause pain. Deep aching pain with gripping, weakness and mechanical symptoms of clicking with pronation-supination can be experienced. The pain is typically described as being sharp and in one spot, frequently accompanied by clicking noises and visually unstable wrist. Though the wrist may show swelling early on, this can quickly subside. Repetitive pronation and supination lead to progressive degenerative changes of the proximal and distal aspects of the horizontal portion of the TFCC. ANATOMY \& PATHOPHYSIOLOGY: TFCC is a constellation of many structures that support the triangular fibrocartilage proper, located between the distal ulna and ulnar carpus, arising from the distal aspect of the sigmoid notch of the radius, inserting in the base of the ulnar styloid process and ulnar carpus via the ulnolunate, ulnotriquetral, and ulnar collateral ligaments. The triangular fibrocartilage is the main ligament that stabilizes the distal radio-ulnar (RU) joint. The distal RU joint is the part of the wrist joint that lies between the two bones of the forearm and allows forearm rotation. The TFCC also functions as a shock-absorber for the wrist, like a meniscus in the knee. When the TFCC is torn, the distal RU joint becomes unstable, which causes the patient pain. TFCC is classified either as Class 1- Traumatic or Class 2 - Degenerative. DIAGNOSTIC TESTING \& CONSIDEERATIONS: Imaging is not very successful at diagnosing TFCC cases. X ray's are negative because there is often no bone damage present during TFCC injuries, and MRI's frequently show a false negative. A thorough physical examination is needed to diagnose TFCC. Immediate examination of the wrist would not present any obvious abnormalities because swelling often recedes quickly. During palpation, the patient would frequently report tenderness along the ulnar side of the wrist, as well as lack range of motion in both supination and pronation, as well as ulnar and radial deviation, An axial load test would need to be performed, which would cause pain on the ulnar side of the wrist if a TFCC tear is present. The patient would also be subjected to a Sharpey's test, which, if a TFCC tear is present, would result in a distinct clicking noise and the ulna translating inferiorly. TREATMENT \& RETURN TO ACTIVITY: Conservative treatment would consist of rest, immobilization in a wrist splint, nonsteroidal anti-inflammatory medication, corticosteroid injections and change in work environment and sport activity. If this fails to resolve pain, physical therapy can then be prescribed. If these conservative treatment options continue to prove unsuccessful, then surgery can be considered. This would usually consist of arthroscopic debridement, total or partial excision, ulnar recession, partial or complete resection of the distal ulna and arthrodesis of the distal radio-ulnar joint followed by an open repair surgery for the TFCC in order to stabilize the distal radio-ulnar joint. 